\title{
Metastatic Non-Small Cell Lung Cancer in the Young: A Retrospective Comparative Analysis
}

Zineb Benbrahim ${ }^{1 *}$, Lamiae Amaadour ${ }^{1}$, Lamiae Boudahna ${ }^{1}$, Naima Abda $^{2}$, Chakib Nejjari², Samia $\quad$, Nawfel Mellas ${ }^{1}$ and Omar El Mesbahi ${ }^{1}$

${ }^{1}$ Medical Oncology department, Hassan II University Hospital, Fez, Morocco

${ }^{2}$ Laboratory of Epidemiology, School of medicine and Pharmacy of Fez, Morocco

\begin{abstract}
Background: Non-small cell lung cancer (NSCLC) in young patients is uncommon and is thought to constitute a distinct oncological entity with characteristic clinicopathological patterns. The objective of our work is to analyze presentation, management and outcome data of NSCLC patients aged less than 45 years and compare them with
\end{abstract} those of patients over 45 years old.

Materials and methods: We retrospectively reviewed all patients treated for metastatic NSCLC over the period of 5 years (2007-2012) at the Medical Oncology Department of Hassan II University Hospital. The clinicopathological characteristics of patients aged less than and over 45 years old were compared and evaluated for prognostic significance regarding outcome.

Results: The data for NSCLC patients (116), of whom 15 were aged $<45$, were retrieved. In comparative analysis, there was a trend for an increased proportion of females with lung cancer among the younger individuals $(56 \%$ in the younger vs $23 \%$ in the older age group, $p=0.008)$. History of smoking was less frequently retrieved in younger patients $(40 \%)$ than patients aged over $45(81.2 \%)(p=0.005)$.No differences were found for hemoptysis, performance status, histology or number of metastatic sites. More patients in the young group $(86.7 \%)$ received biotherapy with platinum based regimen than in older patients $(50.5 \%) ;(p=0.008)$. The mean number of cycles was 2.6 per patient. The Progression free survival (PFS) and overall survival (OS) were not significantly different (median PFS: 6.0 vs 5.0 months; $p=0.9$ and median OS 7.0 vs. 5.0 months, $p=0.14$ ).

Conclusion: This retrospective study failed to present strong evidence that NSCLC among young individuals constitutes a distinct clinicopathological entity. The limited number of younger patients treated here, suggests designing other studies to shed light on this controversial subject.

Keywords: Non-small cell lung cancer; Clinicopathological; Lung cancer; Prognostic features

\section{Background}

In Morocco, according to the Metropolitan Casablanca Region's Register, lung cancer constitutes the leader cause of cancer [1]. Among this population, the incidence of NSCLC diagnosed in the elderly population is high due to the increasing life expectancy.This has been explained by the time required to induce genetic damage leading to cancer [2]. On the other hand, lung cancer occurring in the young is rare. Therefore, few and controversialdata are available regarding clinical characteristics, disease status, treatment modality, and survival of young patients with lung cancer [3]. Conflicting results may be due to the different cutoff ages used when defining the young population, small number of patients enrolled in the studies and genetic differences between the populations studied. In addition, because of a trend to increasing incidence in this population, the need of its characterization becomes more pressing. The aim of this study was to document the incidence, clinico-pathological characteristics, treatment outcomes and prognostic features of lung cancer patients between 15 and 44 years of age living in Morocco. A comparison with lung cancer patients of any age from the same population was also made.

\section{Materials and Methods}

This study retrospectively reviewed patients with NSCLCs who presented between April 2007 and December 2012 at the Department ofMedical Oncology at Hassan II University Hospital in Fez, Morocco.

Eligible patients had histological diagnosis of NSCLC classified as stage IV according to the TNM staging system. Exclusion criteria were previous treatment for the current cancer.The patients were further classified into two groups:young adults $<45$ and patients aged $\geq 45$ years old.

Standardized data relating to age, gender, smoking habits, presenting symptoms, performance status, histology, stage at diagnosis, biological perturbations, number of metastatic sites, treatment (chemotherapy, best supportive care), and outcome were collected and analyzed.

Tumor responses were evaluated according to the RECIST criteria. Progression-free survival (PFS) was defined as the time elapsed between treatment initiation and tumor progression or death from any cause. Overall survival (OS) was defined as the time from the diagnosis to death.

For statistical analysis, the Kaplan-Meier method with a Logrank test was used for survival analysis. The Cox proportional hazards model

*Corresponding author: Zineb Benbrahim, Medical Oncology department Hassan II University Hospital, BP: 8743 Atlas Fès. Morocco, Tel: 002126627840 88; E-mail: zineb247@hotmail.com

Received November 25, 2013; Accepted April 02, 2014; Published April 05, 2014

Citation: Benbrahim Z, Amaadour L, Boudahna L, Abda N, Nejjari C, et al. (2014) Metastatic Non-Small Cell Lung Cancer in the Young: A Retrospective Comparative Analysis. J Cancer Sci Ther 6: 148-150. doi:10.4172/1948-5956.1000263

Copyright: (C) 2014 Benbrahim Z, et al. This is an open-access article distributed under the terms of the Creative Commons Attribution License, which permits unrestricted use, distribution, and reproduction in any medium, provided the original author and source are credited. 
was used for multivariate analysis. Frequencies were compared using the chi-square test for qualitative variables. The Fisher exact test was used when the expected frequency of one of the variables was less than 5. A P-value of -0.05 was considered to be statistically significant. All statistical analyses were performed using the SPSS statistical software package.

\section{Results}

From 2007 to 2012, 116 patients with metastatic NSCLC were registered: 15 (12.9\%) were aged lessthan 45 years.

In the entire study, the mean age was $57.06 \pm 10.7$ years ( 40.6 in the young group vs 59.5 in the older group).19 cases (16.4\%) of NSCLC occurred in females and $97(83.6 \%)$ in males. There was a trend for an increased proportion of females with lung cancer among the younger individuals ( $56 \%$ in the younger vs $23 \%$ in the older age group, $\mathrm{p}=0.008$ ).

For smoking history, it was less frequently retrieved in younger patients (40\%) than patients aged over $45(81.2 \%)(\mathrm{p}=0.005) .73 .3 \%$ of young patients had performance status $\leq 1$ compared to $56.4 \%$ in older group. However this difference was not statistically significant $(\mathrm{p}=0.1)$

The most commonly reportedsymptoms were thoracic pain (60\%), dyspnea (51\%), cough (45\%) and hemoptysis (34\%). In comparativeanalysis, there was no significant statistical difference regarding symptoms between the two groups.

Adenocarcinoma was the leading celltype in this studyaccounting for $50 \%$ of tumours. It occurred more frequently in both young $(\mathrm{N}=9)$ and older groups $(\mathrm{N}=49)$. Squamous cell carcinoma was less frequently diagnosed in the young population $(\mathrm{N}=2)$ than in the older one $(\mathrm{N}=27)$.

Biologically, anemia was noted in about two thirds of patients in both groups ( $57.1 \%$ vs $58.2 \%$; $=0.4$ ). No high level of calcemia was noted in young group versus $9.9 \%$ in older group $(\mathrm{p}=0.2)$. Younger patients had numerically more hypoalbuminemia (66.7\%) than elderly patients $(42.4 \%)$, however it didn't reach statistical significance $(\mathrm{P}=0.1)$.

No differences were found for number of metastatic sites or type oftreatment provided.Regarding the treatment, there were more patients who received doublet of chemotherapy comprising cisplatin ( $86.7 \%$ vs $50.5 \%$; $\mathrm{p}=0.004$ ). The median number of administered cycles had been two per patient.

The median progression free survival for the group of young patients was 6.0 months ( $95 \%$ CI: 4.58-7.42), compared to 5.0 months (95\% CI, 3.97- 6.02) for the older individuals. However, the survival difference between the two groups was not significant in Logrank test analysis $(p=0.9)$ (Figure 1 ).

Three cases of death were noted in the young versus 28 cases in the older group. The median overall survival for the group of patients $<45$ years was 7.0 months (95\% CI: $5.40-8.50$ ), compared to 5.0 months (95\% CI, 3.6-6.3) for the group $>45$ years. Nevertheless, the survival difference between the two groups was not significant in Logrank test analysis $(p=0.14)$ (Figure 2).

The prognostic value of the hypoalbuminemia was established in both groups. Hence, in the young group, the overall survival was 72.0 months in the normo-albuminemia subgroup versus 4.0 months in hypoalbuminemia subgroup $(\mathrm{p}=0.02)$. Otherwise, in the older group, the overall survival was 9.3 months in the normo-albuminemia subgroup versus 4.0 months in the hypoalbuminemia subgroup $(\mathrm{p}=0.01)$.

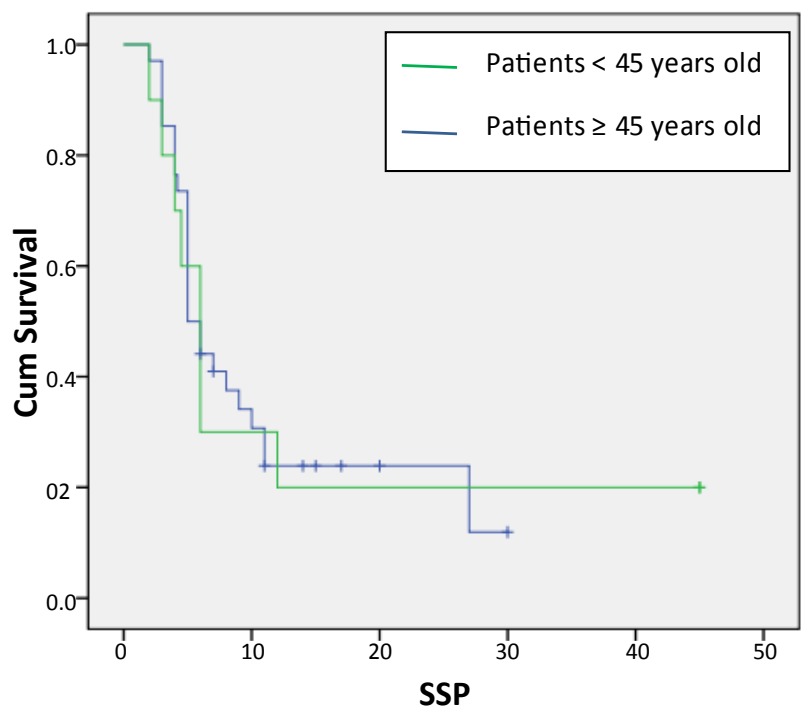

Figure 1: Progression Free Survival according to the age of patients ( $\geq 45$ vs $<45$ years old).

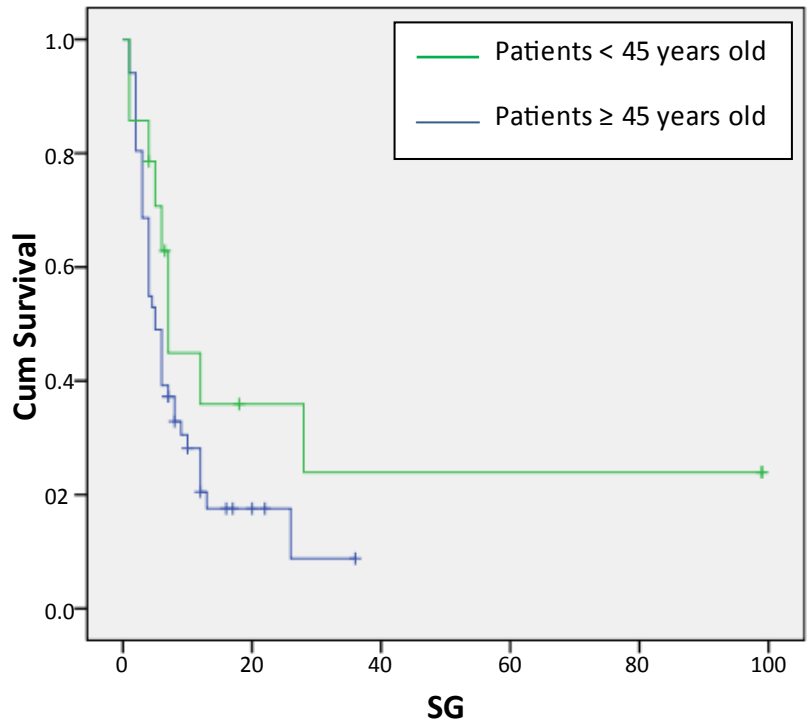

Figure 2: Overall Survival according to the age of patients ( $\geq 45$ vs $<45$ years old).

\section{Discussion}

Lung cancer generally occurs in people between 50 and 80 years old [4]. However, it has become less rare in patients younger than 45 years over the last decades [5-6]. In the literature, conflicting data about young patients' clinical characteristics and prognoses are recorded[5-9]. These differences might result from different cutoff ages when defining young patients. In this paper, we defined lung cancer in young patients as patients with age under 45 years as in most publications retrieved in the literature[10-12].

In order to determine whether younger patients with NSCLC 
have different clinical and prognostic features than older patients we conducted the present study that found several interesting characteristics of young patients with lung cancer.

First of all, predominance of male patients in the group $>45$ years old compared to the younger group (Male/Female ratio 6.7 vs 1.5 ) indicates that in younger patients, more female are suffering from lung cancer when compared with older group. This ascertainment may probably due to increased smoking in the female population especially in the teenage years and the different physiopathology and biologic characteristics in lung cancer occurring in the young.

Smoking history was less frequently retrieved in younger patients. This is related to the time that cancer takes to develop after starting to smoke leading to the occurrence of more smoking related carcinoma in the older group.

Furthermore, our results on the presenting symptomatology of the disease are in contrast with those reported by Bourke et al. [13] who found statistically higher rates of cough and chest pain among young patients compared to older individuals.

Regarding histologic features, adenocarcinoma accounts for most of the NSCLC in young patients in the literature [5-8,14-15]. In our study, the percentage of adenocarcinoma was higher in the younger group ( $60 \%$ vs $48.5 \%)$ without statistical significance $(p=0.2)$.

In addition, young patients seem to have better performance status at diagnosis and lower number ofcomorbidities. Consequently, more aggressive treatment modalities are feasible in this population [12]. On the other hand, lung cancer in young patients has worse prognosis because of its aggressive biological behavior [6]. This difference in survival was not demonstrated in this study probably because of limited number of younger patients treated here.

Finally, although this is the first analysis of lung cancer in patients younger than 45 years in Morocco, this paper has some limitations. The main one is its retrospective nature. Besides, Epidermal Growth Factor Receptor mutations were not searched in this study and patients did not benefit from Tyrosine kinase inhibitors targeting these mutations. Therefore,others prospective studies are needed to define the genetic susceptibility for lung cancer inthe younger patients.

\section{Conclusion}

This was the first analysis in Morocco and one of the fewer papers published in the world that were interested in lung cancer among the young population. Some different epidemiological and clinical features were determined such as an increased proportion of female, predominance of non-smoker patients and adenocarcinoma subtype and the ability to receive a doublet of a platin based chemotherapy. However, this paper failed to present strong evidence that NSCLC among young individuals had a different prognostic than older individuals probably for lack of power in this study. The limited number of younger patients treated here, suggests designing other studies to shed light on this controversial subject.

\section{References}

1. http://www.contrelecancer.ma/en/media/2012/05/26/le-nouveau-registre-descancers-de-la-region-du-grand-casablanca-est-paru/

2. Mauri D1, Pentheroudakis G, Bafaloukos D, Pectasides D, Samantas E, et al. (2006) Non-smallcelllung cancer in the young: aretrospectiveanalysis of diagnosis, management and outcome data. AnticancerRes 26: 3175-3181.

3. Strand TE, Malayeri C, Eskonsipo PK, Grimsrud TK, Norstein J, et al. (2004) Adolescent smoking and trends in lung cancer incidence among young adults in Norway 1954- 1998. Cancer Causes Control 15: 27-33.

4. Ramalingam S1, Pawlish K, Gadgeel S, Demers R, Kalemkerian GP (1998) Lung cancer in young patients: analysis of a Surveillance, Epidemiology, and End Resultsdatabase. J Clin Oncol 16: 651-657.

5. Mauri D1, Pentheroudakis G, Bafaloukos D, Pectasides D, Samantas E, et al. (2006) Non-small cell lung cancer in the young: aretrospectiveanalysis of diagnosis, management and outcome data. Anticancer Res 26: 3175-3181.

6. Yang P1, Bamlet WR, Ebbert JO, Taylor WR, de Andrade M (2004) Glutathione pathway genes and lung cancer risk in young and old populations. Carcinogenesis 25: 1935-1944.

7. Liam CK, Lim KH, Wong CM (2002) Non-small cell lung cancer in very young and veryold Malaysian patients. Chest 121: 309-310.

8. Skarin AT1, Herbst RS, Leong TL, Bailey A, Sugarbaker D (2001) Lung cancer in patients underage 40. Lung Cancer 32: 255-264.

9. Radzikowska E1, Roszkowski K, GÅ,az P (2001) Lung cancer in patients under 50 years old. Lung Cancer 33: 203-211.

10. Capewell S1, Wathen CG, Sankaran R, Sudlow MF (1992) Lung cancer in young patients. Respir Med 86: 499-502.

11. Kreuzer M1, Kreienbrock L, Gerken M, Heinrich J, Bruske-Hohlfeld I, et al (1998) Riskfactors for lung cancer in young adults. Am J Epidemiol 147: 10281037.

12. Zhang J1, Chen SF, Zhen Y, Xiang J, Wu C, et al. (2010) Multicenter analysis of lung cancer patients younger than 45 years in Shanghai. Cancer 116: 3656 3662 .

13. Birim O1, Zuydendorp HM, Maat AP, Kappetein AP, Eijkemans MJ, et al. (2003) Lung resection for non-small-cell lung cancer in patients olderthan 70: mortality, morbidity, and late survival compared with the general population. Ann Thorac Surg 76: 1796-1801.

14. Radzikowska $\mathrm{E} 1$, Roszkowski K, GÅ,az P (2001) Lung cancer in patients under 50 years old. Lung Cancer 33: 203-211.

15. Minami H1, Yoshimura M, Matsuoka H, Toshihiko S, Tsubota N (2001) Lung cancer treatedsurgically in patients $<50$ years of age. Chest $120: 32-36$. 